\title{
Dynamic Modal Correlation of an Automotive Rear Subframe, with Particular Reference to the Modelling of Welded Joints
}

\author{
Vincenzo Rotondella, ${ }^{1}$ Andrea Merulla, ${ }^{2}$ Andrea Baldini, ${ }^{2}$ and Sara Mantovani ${ }^{1}$ \\ ${ }^{1}$ MilleChili Lab, Department of Engineering "Enzo Ferrari”, Università degli Studi di Modena e Reggio Emilia, \\ Via Vivarelli 10, 41124 Modena, Italy \\ ${ }^{2}$ Ferrari S.p.A, Via Abetone Inferiore 4, 41053 Modena, Italy \\ Correspondence should be addressed to Sara Mantovani; sara.mantovani@unimore.it
}

Received 20 December 2016; Revised 27 February 2017; Accepted 8 March 2017; Published 3 April 2017

Academic Editor: Marc Thomas

Copyright ( 2017 Vincenzo Rotondella et al. This is an open access article distributed under the Creative Commons Attribution License, which permits unrestricted use, distribution, and reproduction in any medium, provided the original work is properly cited.

\begin{abstract}
This paper presents a comparison between the experimental investigation and the Finite Element (FE) modal analysis of an automotive rear subframe. A modal correlation between the experimental data and the forecasts is performed. The present numerical model constitutes a predictive methodology able to forecast the experimental dynamic behaviour of the structure. The actual structure is excited with impact hammers and the modal response of the subframe is collected and evaluated by the PolyMAX algorithm. Both the FE model and the structural performance of the subframe are defined according to the Ferrari S.p.A. internal regulations. In addition, a novel modelling technique for welded joints is proposed that represents an extension of ACM2 approach, formulated for spot weld joints in dynamic analysis. Therefore, the Modal Assurance Criterion (MAC) is considered the optimal comparison index for the numerical-experimental correlation. In conclusion, a good numerical-experimental agreement from $50 \mathrm{~Hz}$ up to $500 \mathrm{~Hz}$ has been achieved by monitoring various dynamic parameters such as the natural frequencies, the mode shapes, and frequency response functions (FRFs) of the structure that represent a validation of this FE model for structural dynamic applications.
\end{abstract}

\section{Introduction}

The employment of Finite Element (FE) models to predict the dynamic properties of a vehicle has continuously become more important in modern automotive industries. Whenever there is a new design or modification of an existing one, the structural dynamic properties of the car should be examined to fulfil some criteria proposed by the industry itself before the product can be launched on the market.

The traditional methodology for evaluating the structural dynamic properties of a vehicle is to perform various dynamic tests on prototypes of the product and to demonstrate their capacity to withstand these tests. Until the experimental results show that the prototypes can comply with the relevant criteria, the component has to be redesigned and another design-test loop must be followed. In this designtest-redesign loop, the higher percentage of time and financial resources is spent in producing prototypes and performing tests.

With the growing capabilities of computing techniques, and the strength of the competition between companies, FE model predictions are used more and more frequently to substitute practical dynamic test data. Furthermore, the FE modelling technique may also be used to predict the dynamic response of structures when working beyond a limit situation that makes the simulations by experiment extremely difficult, if not impossible. All of these results depend on the accuracy of FE model predictions.The validation of FE models and their capability to predict the dynamic behaviour of the structures are crucial topics for industrial purposes and especially for aerospace and automotive applications.

In 1990s, Baker [1], Imregun and Visser [2], and Friswell and Mottershead [3] performed a complete review of different correlation methods and validation criteria for structural 
dynamic behaviour, in order to investigate the methodologies that might improve the prediction capability of FE models.

Brughmans et al. [4] focused on test-analysis correlation for vibroacoustic application, integrating methods and algorithms in a high performance computational environment. In their research, Jambovane et al. [5] employed experimental modal analysis to validate FE model of an engine oil pan. The correlation between experimental and numerical modal analyses on panels has been performed by Siano et al. [6] and Splendi et al. [7] in order to verify the FE model reliability for Noise Vibration Harshness (NVH) applications.

Schedlinski et al. [8] presented the validation of an FE model of a Body-in-White using the computational modal updating procedure to improve the quality and prediction of the model.

In addition to the previous works, $[9,10]$ confirmed the experimental modal analysis as a fundamental requirement for the validation of a FE model, built to perform dynamic analysis. The present paper is based on original experimental data, and it describes a detailed methodology that aims to investigate the capability and reliability of a structural FE model for NVH applications. The present research is performed at MilleChili laboratory from the University of Modena and Reggio Emilia and in collaboration with Ferrari S.p.A.

\section{Materials and Methods}

The analyzed structure is an aluminium rear subframe that can be disassembled from the chassis; it is made of eleven extruded beams, plates, gussets, and two casting components, as shown in Figure 1. There are eight points on each side that allow the connection of the subframe to the chassis. The suspension mounting points are located on the casting component. This is a prototype component of the chassis and the different parts are jointed together through metal inert gas (MIG) welding. MIG is one of the most widely used forms of welding in industry, and it is considered one of the easiest forms of welding to learn. The total weight of the rear subframe is $26.3 \mathrm{~kg}$, including the testing devices (e.g., suspension "biscuits" and gearbox mounting "clocks") and the suspension mounting bolts.

2.1. Experimental Modal Analysis (EMA). As pointed out by Schwarz and Richardson [11], natural modes of vibrations are inherent properties of a structure. Modes or resonances are defined by physical properties (mass, stiffness, and damping) and boundary conditions of the component. The modal properties of a structure are natural frequencies, modal damping, and mode shapes. A modal testing could be summarized in two main different phases:

(i) Structure test to obtain FRF measurements

(ii) FRF curve fitting to extract experimental modal parameters

A fast and convenient way to find the modes of a structure is impact testing. It was developed in the 1970's and it has become the most popular modal testing used for evaluating

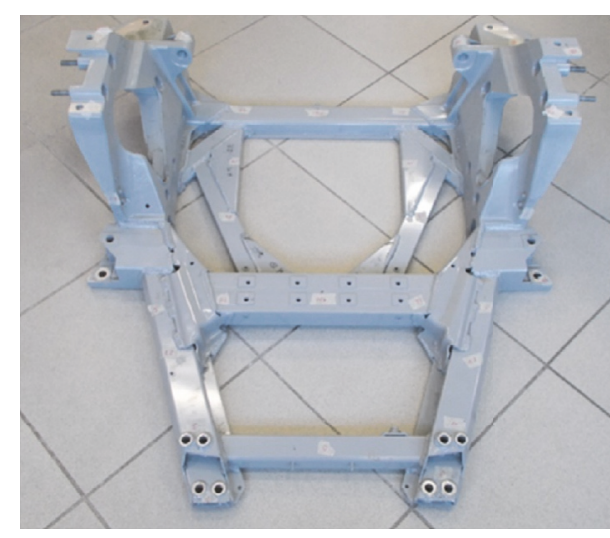

FIgURE 1: Aluminium rear subframe.

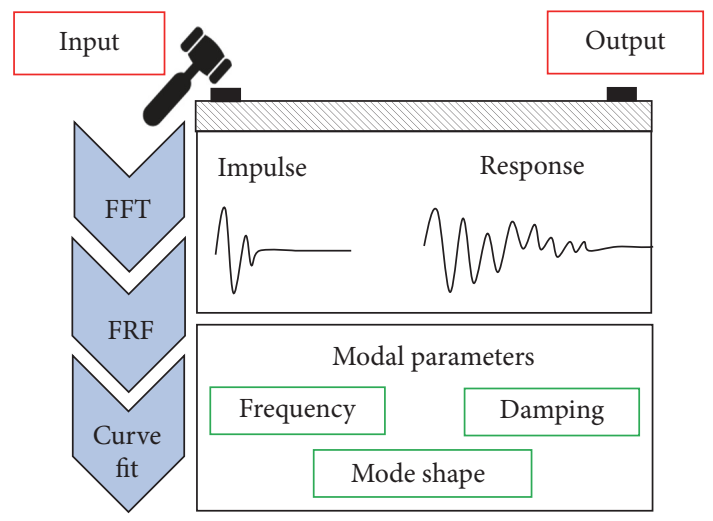

FIGURE 2: EMA through impact test.

the modal properties of a structure. The equipment required to perform an impact test is

(i) Impact hammer

(ii) Accelerometers

(iii) FFT analyzer

(iv) Postprocessing modal software

Figure 2 shows the whole process of EMA through impact test.

The modal parameter estimation is obtained from a set of frequency response function (FRF) measurements. The FRF, as explained in [11], "describes the input-output relationship between two points on a structure as a function of frequency." The basic formula of FRF is

$$
H(\omega)=\frac{Y(\omega)}{X(\omega)},
$$

where

(i) $H(\omega)$ is the frequency response function,

(ii) $Y(\omega)$ is the output of the system in the frequency domain,

(iii) $X(\omega)$ is the input of the system in the frequency domain. 


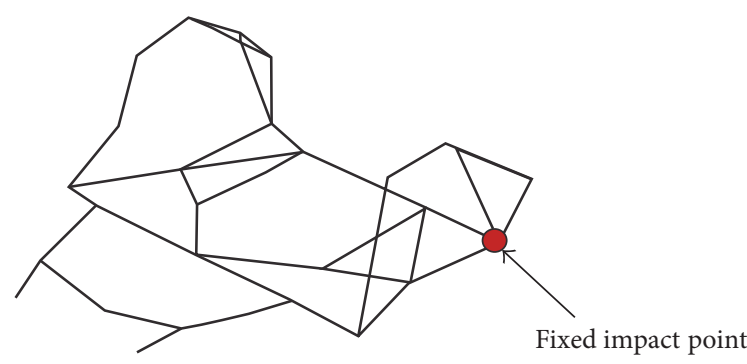

FIgURE 3: Test geometry of the rear subframe.

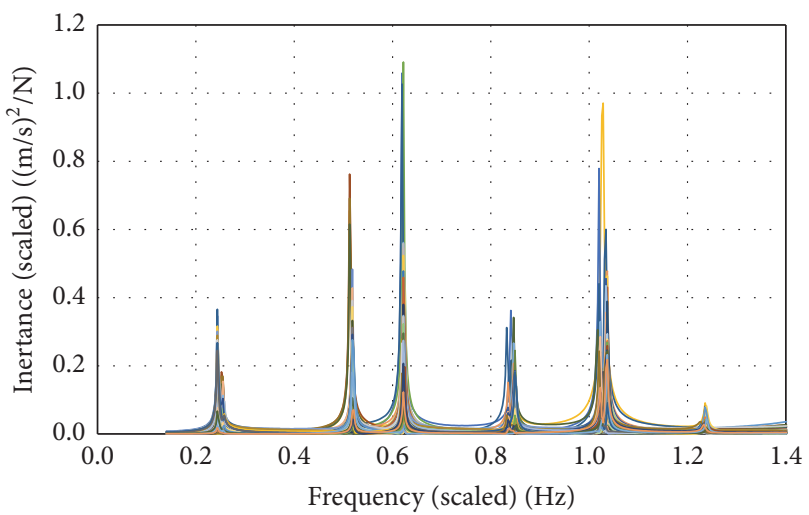

FIGURE 4: Plots of FRFs of the 35 reference points.

The modal test was performed at the NVH experimental department of Ferrari S.p.A. The structure was suspended by four soft elastic bungees in order to simulate the freefree condition; this condition means that the structure is not connected to the ground at any of its coordinates and it is, in effect, freely suspended in space. In this condition, the structure will exhibit rigid body modes, which are determined merely by its mass and inertia properties. It had been verified in a pretest phase that the suspension system did not interfere with the modes of vibration of the structure. A single impact point on the lower side of the left casting in the $Z$-direction was used during the test. The responses were measured in 35 positions, using three-axis accelerometers. The geometry (or wireframe) of the experimental setup is shown in Figure 3, where the red dot indicates the location of the impact point. The software used for impact testing is LMS Test.Lab.

Parameter estimation techniques for modal analysis are based on the extraction of natural frequency, the damping, and the mode shapes from the experimental data, which is in a processed form such as frequency response functions (FRFs). Figure 4 shows the FRFs collected from the structure during the impact test. The considered FRFs are the socalled inertance or receptance, because they measure the acceleration response of the structure at an output point, per unit of excitation force at an input point. The numerical values presented in this paper have been scaled by an arbitrary factor for secrecy reasons.

The estimation method used in this phase was PolyMAX (Peeters et al. [12]), which is available in LMS Test.Lab. As described in [12], the PolyMAX method employs measured FRFs as primary data and it is called the $z$-domain method (i.e., a frequency-domain model is derived from a discretetime model). The poles and modal participation factors could be retrieved following the right matrix-fraction model:

$$
[H(\omega)]=\sum_{r=0}^{p} z^{r}\left[\beta_{r}\right] \cdot\left(\sum_{r=0}^{p} z^{r}\left[\alpha_{r}\right]\right)^{-1},
$$

where

(i) $[H(\omega)]$ is the matrix containing the FRFs between all $m$ inputs and all $l$ outputs,

(ii) $z=e^{-j w \Delta t}$ is the frequency-domain model,

(iii) $\left[\alpha_{r}\right]$ and $\left[\beta_{r}\right]$ are the denominator and the numerator matrix polynomial coefficients,

(iv) $p$ is modal order.

Once the coefficients $\left[\alpha_{r}\right]$ and $\left[\beta_{r}\right]$ are determined solving (2), it is possible to calculate the poles $\lambda_{i}, \lambda_{i}^{*}$, which are related to the eigenfrequencies $\omega_{i}$ and to the damping ratio $\xi_{i}$ as follows:

$$
\lambda_{i}, \lambda_{i}^{*}=\xi_{i} \omega_{i} \pm \sqrt{1-\xi_{i}^{2}} \omega_{i} .
$$

The mode shapes could be determined by considering pole-residue model:

$$
\begin{aligned}
& {[H(\omega)]} \\
& \quad=\sum_{i=1}^{n} \frac{\left\{v_{i}\right\}\left\langle l_{i}^{T}\right\rangle}{j \omega-\lambda_{i}}+\frac{\left\{v_{i}^{*}\right\}\left\langle l_{i}^{T *}\right\rangle}{j \omega-\lambda_{i}^{*}}-\frac{[\mathrm{LR}]}{\omega^{2}}+[\mathrm{UR}],
\end{aligned}
$$

where

(i) $[H(\omega)]$ is the matrix containing the FRFs between all $m$ inputs and all $l$ outputs,

(ii) $n$ is the number of modes,

(iii) $\left\{v_{i}\right\}$ and $\left\{v_{i}^{*}\right\}$ are the complex conjugate mode shapes,

(iv) $\left\langle l_{i}^{T}\right\rangle$ and $\left\langle l_{i}^{T *}\right\rangle$ are the complex conjugate modal participation factors,

(v) $\lambda_{i}$ and $\lambda_{i}^{*}$ are the complex conjugate poles,

(vi) [LR] and [UR] are the lower and upper residuals which model the influence of the out-of-band modes.

Estimated poles are calculated from (2) and (3) and the results of this operation are presented in a so-called stabilization diagram. The interpretation of the stabilization diagram yields to a set of complex poles and participation factors, which are inserted in (4). Solving (4) in a linear leastsquares sense, it is possible to retrieve the mode shape for each eigenfrequency.

Figure 5 shows the stabilization diagram employed to determine the modal parameters using PolyMAX method inside LMS Test.Lab software. Table 1 lists the natural frequencies, from $50 \mathrm{~Hz}$ up to $500 \mathrm{~Hz}$, scaled, which was identified through EMA. 


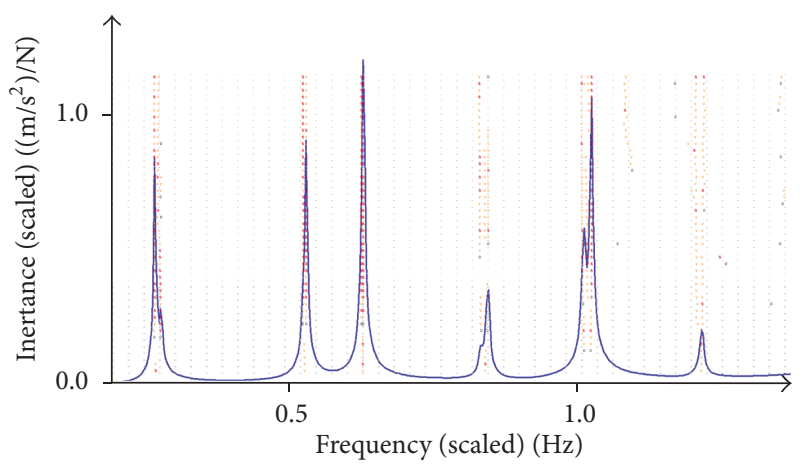

FIgURE 5: Stabilization diagram for PolyMAX method; the blue curve represents the sum of FRFs.

TABLE 1: Natural frequencies (scaled) from EMA.

\begin{tabular}{lcc}
\hline Mode & $\begin{array}{c}\text { Frequency } \\
\text { (scaled) }[\mathrm{Hz}]\end{array}$ & $\begin{array}{c}\text { Damping ratio } \\
(\%)\end{array}$ \\
\hline 1 & 0.244 & 0.20 \\
2 & 0.254 & 0.35 \\
3 & 0.519 & 0.04 \\
4 & 0.623 & 0.08 \\
5 & 0.834 & 0.08 \\
6 & 0.849 & 0.03 \\
7 & 1.023 & 0.09 \\
8 & 1.037 & 0.07 \\
9 & 1.236 & 0.13 \\
\hline
\end{tabular}

2.2. Finite Element Modal Analysis. The FE modelling techniques used for this study followed the internal criteria of the CAE Department of Ferrari S.p.A.

The extruded beams were modelled according to shell formulation using CQUAD4 and CTRIA3 elements. The castings were modelled with tridimensional elements; secondorder CTETRA were used in order to compensate the spatial discretization. The description of the Finite Element model of welded joints is widely discussed in the next paragraph. Testing devices (e.g., suspension "biscuits" and gearbox mounting "clocks") were added to the FE model in order to accurately reproduce the testing setup. They were connected to the structure through CBAR elements. Nonstructural mass NSML1 was applied to align the numerical mass to the weight of the physically tested structure, taking into account the mass added from coating process.

Figure 6 shows the full three-dimensional view of the FE model of the rear subframe.

2.3. FE Modelling Technique for Welded Joints. The FE approach for modelling the welded joints is a relevant research topic for the present study. There are two different techniques for welding models: for stress analysis and for stiffness-based analysis. Obviously, the choice of the modelling technique depends on the aim of FE analysis performed. In literature [13], different methods for modelling the welded joints are proposed, mainly addressing stress

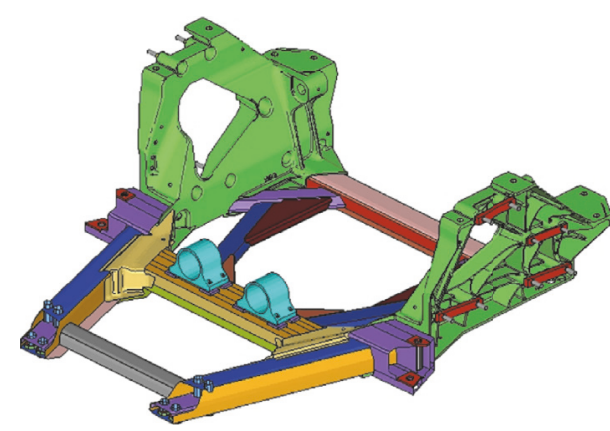

FIGURE 6: FE model of rear subframe.

analysis. Welded joints should be modelled in cases where stress is influenced by bending behaviour, when it is not easy to distinguish the nonlinear stress, caused from the notch to the welding foot, by stress concentrations effects that arise from geometric irregularities. In these cases, the stiffness of the welded joint section should be taken into account and the welds have to be modelled using several techniques. Although the stress field is detailed, the evaluation of the stiffness is not necessary accurate; for modal analysis this is a mandatory parameter; then this approach is not advised for this activity. Stiffness-based models of welding require an accurate representation of the stiffness (and the mass) of the joints. Furthermore, one fundamental requirement is a limited time spent in the FE model assessment. In the past, very simple models of welded joints have been used extensively in automotive industry; they consist of elastic or rigid one-dimensional elements or coincident nodes. The disadvantage of these models is the inadequacy to represent the behaviour of the welding and the underestimation of the stiffness. For modal and static stiffness analysis, a brick model by $\mathrm{Pal}$ and Cronin [14] was proposed. In this technique, a brick element was used to represent the spot weld and the connection between the shell plates and the solid was performed through rigid elements. A very accurate stiffness was guaranteed; however a mesh congruence between shell and brick elements was necessary and thus a high time of model setup was required. To overcome this problem, Backhans and Cedas [15] proposed the ACM1 method that allowed the connection between noncongruent shell meshes through rigid beam forming an umbrella shape. In recent years, the three-dimensional approach for welding is becoming more commonly employed in FE models for industrial analysis because they well represent the stiffness, the geometry, and also the mass of the connection. Heiserer et al. [16] proposed the model known as ACM2 for spot welds. As described in [17], the model consists of a brick element connecting the upper and lower plates via RBE3 elements. The RBE3 element distributes the applied loads throughout the model. Forces and moments applied to the brick nodes are distributed to the shell nodes in a way that depends on the RBE3 geometry and weight factors assigned to the shell nodes. The weights are the values assumed by the shape function corresponding to each shell node at the location of the brick node. The force acting on the brick node can be transferred to the 


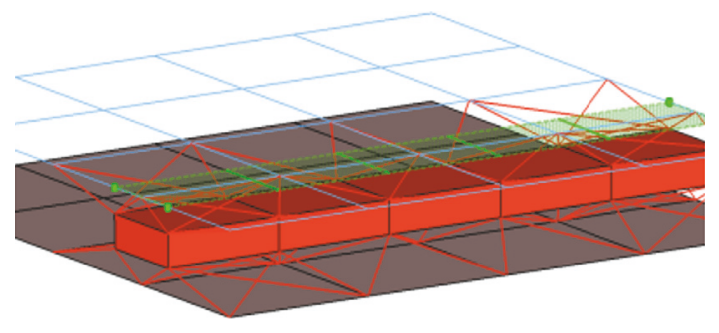

FIGURE 7: Details of ACM2 extended method: CHEXA for the body of the welded joint and RBE2/RBE3 for the head.
TABLE 2: Natural frequencies (scaled) from FEA.

\begin{tabular}{lc}
\hline Mode & Frequency (scaled) $[\mathrm{Hz}]$ \\
\hline 7 & 0.244 \\
8 & 0.252 \\
9 & 0.503 \\
10 & 0.612 \\
11 & 0.817 \\
12 & 0.833 \\
13 & 1.000 \\
14 & 1.019 \\
15 & 1.234 \\
\hline
\end{tabular}

2.4. Modal Correlation. The correlation phase is focused on comparing, understanding, and evaluating the correlation between test and FE data. A modal based index, which is used for comparing experimental and numerical modal shapes, is Modal Assurance Criterion (MAC). MAC was originally developed for orthogonality check and, in the late 1970s, was proposed by Allemang and Brown [18] as a correlation coefficient for modal analysis. The function of the MAC [19] is to provide a measure of consistency between one modal and another reference modal vector. It is defined as a scalar constant which takes on values from zero, representing no consistent correspondence, to one, representing fully consistent correspondence. The MAC value between experimental and numerical FE modal vector is obtained by the following equation:

$$
\begin{aligned}
& \operatorname{MAC}\left(\{\Phi\}_{\text {test }},\{\Phi\}_{\mathrm{FE}}\right) \\
& =\frac{\left|\{\Phi\}_{\text {test }}^{T} \cdot\{\Phi\}_{\mathrm{FE}}\right|^{2}}{\left(\{\Phi\}_{\text {test }}^{T} \cdot\{\Phi\}_{\text {test }}\right) \cdot\left(\{\Phi\}_{\mathrm{FE}}^{T} \cdot\{\Phi\}_{\mathrm{FE}}\right)},
\end{aligned}
$$

where

(i) $\{\Phi\}_{\text {test }}$ is the modal vector of a EMA modal shape,

(ii) $\{\Phi\}_{\text {test }}^{T}$ is the transpose of $\{\Phi\}_{\text {test }}$,

(iii) $\{\Phi\}_{\mathrm{FE}}$ is the modal vector of a FEA modal shape,

(iv) $\{\Phi\}_{\mathrm{FE}}^{T}$ is the transpose of $\{\Phi\}_{\mathrm{FE}}$.

Generally, values of MAC should be above 0.7 for representing a good correlation. It is also worth noting that every degree of freedom (DOF) gives a contribution to the MAC index. So, relatively considerable in-phase displacements of a DOF give a positive contribution to the correlation; relatively considerable out-of-phase displacements give a negative contribution; and relatively small displacements give less important contributions.

\section{Results and Discussion}

The correlation results have been evaluated comparing the natural frequency values and the error percentages, the mode shapes, and the MAC matrix.

Plotting the FEA-EMA values of natural frequencies, the theoretical best fit is represented by a 45 -degree line. The activity and FEA results will refer to it. 
TABLE 3: Comparison between natural frequencies (scaled) from FEA using different methods.

\begin{tabular}{lccc}
\hline Mode & $\begin{array}{c}\text { ACM2 method } \\
\text { Scaled freq. }[\mathrm{Hz}]\end{array}$ & $\begin{array}{c}\text { Rigid elements method } \\
\text { Scaled freq. [Hz] }\end{array}$ & Difference (\%) \\
\hline 7 & 0.244 & 0.237 & 2.742 \\
8 & 0.252 & 0.249 & 1.297 \\
9 & 0.503 & 0.500 & 0.609 \\
10 & 0.612 & 0.617 & -0.896 \\
11 & 0.817 & 0.787 & 3.630 \\
12 & 0.833 & 0.820 & 1.610 \\
13 & 1.000 & 0.978 & 2.193 \\
14 & 1.019 & 1.019 & 0.029 \\
15 & 1.234 & 1.226 & 0.655 \\
\hline
\end{tabular}

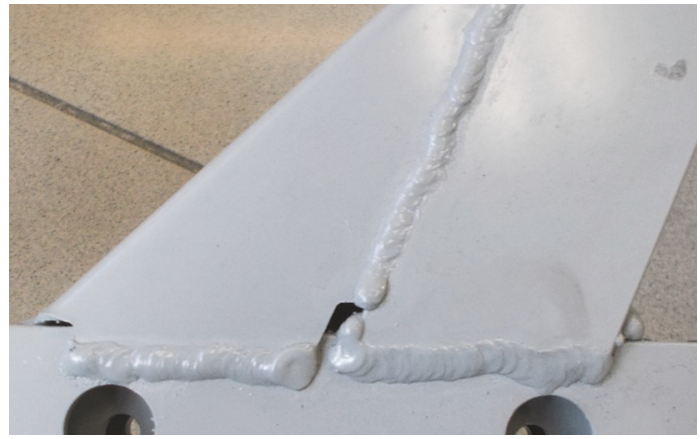

(a)

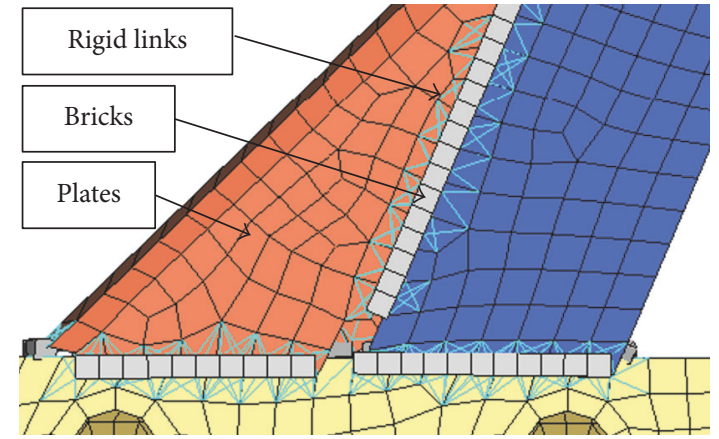

(b)

FIGURE 8: Details of MIG welding on the structure: (a) the actual welded joint, (b) the ACM2 approach.

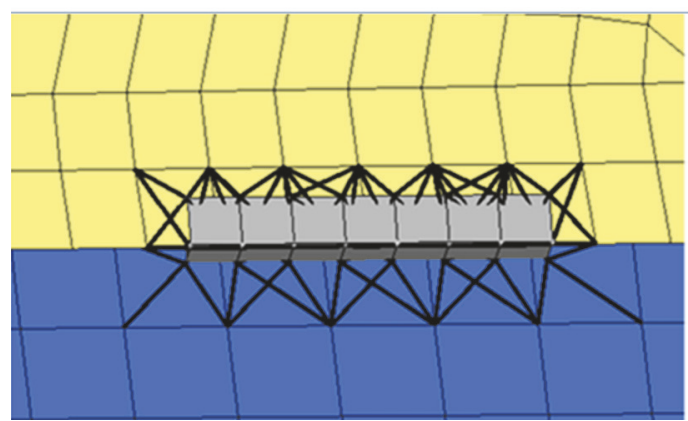

(a)

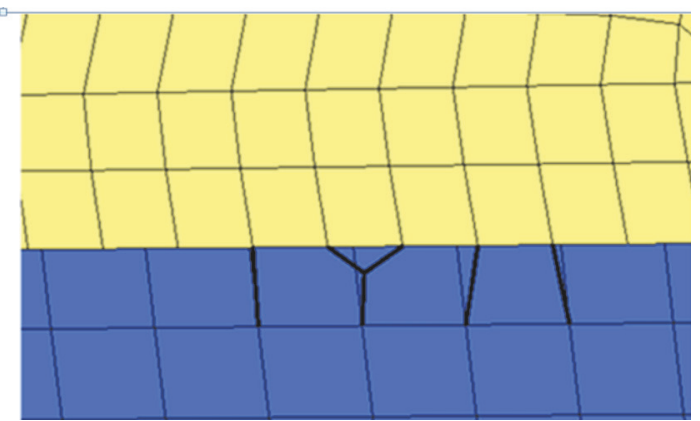

(b)

FIGURE 9: Details of MIG welding models: (a) the ACM2 approach, (b) the rigid elements approach.

natural frequency plot in Figure 10 exhibits an acceptable correlation, in which the frequency couples constitute a line very close to the reference one.

As pointed out in Figure 11, analyzing every single mode, the absolute percentage error between experimental and numerical eigenfrequency is less than 4 percent. The low percentage error represents a simple index to verify the correlation in automotive applications.

Figure 12 shows a numerical and experimental comparison among the deformed shape of the first modes couple. In particular, it corresponds to the first global torsional mode of the structure (EMA scaled frequency is 0.24 and FEA scaled normalized frequency is 0.244 ).
In addition, to a simple visual analysis of each mode shape, a comparison based on MAC values has been performed in order to deeply investigate the correlation achieved. Figure 13 shows a good correlation between EMA and FEA frequencies because the values on the main diagonal of the MAC matrix are greater than 0.7 . This value represents a reliable MAC index for a satisfying correlation for complex structures, as described also in $[6,8]$. Only the fifth mode pair (EMA Mode\#5, FEA Mode\#11) shows a MAC value on the main diagonal of the matrix that is lower than 0.7. The reason of this lack of correlation in terms of MAC could be found in the impact test execution. In Figure 5, the blue curve 


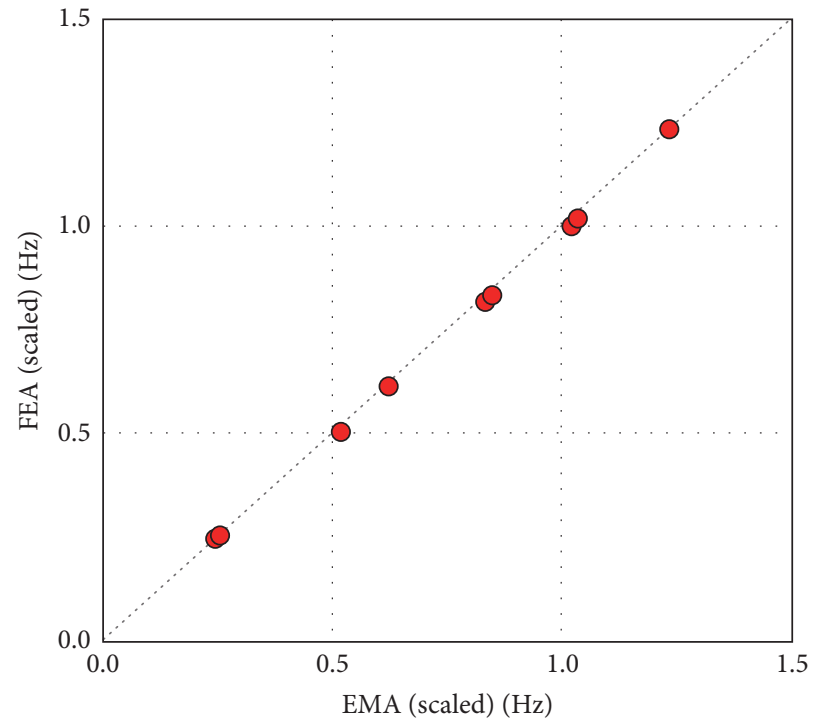

FIGURE 10: Natural frequency pairs plot.

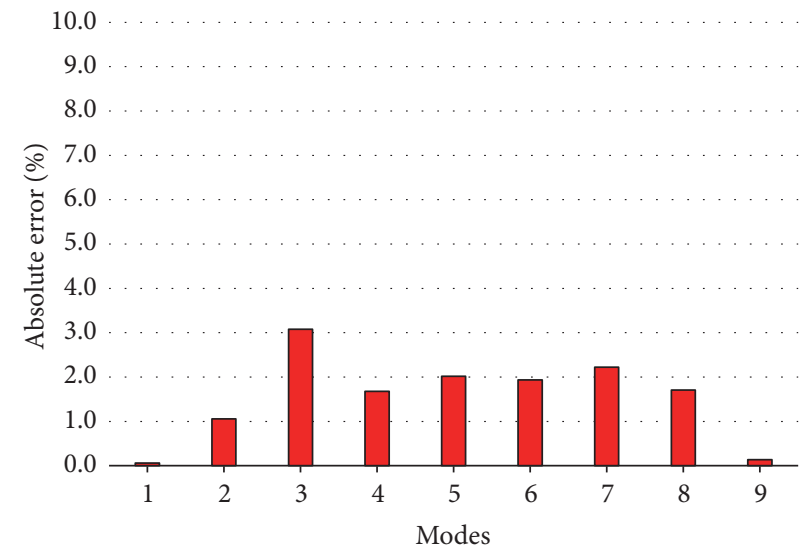

FIGURE 11: Absolute percentage error histogram.

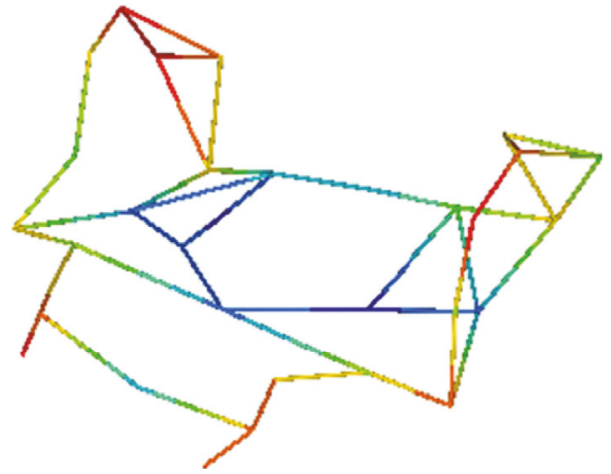

(a)

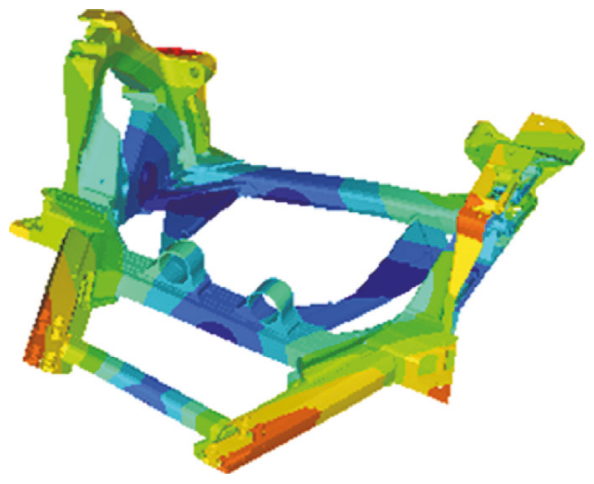

(b)

FIGURE 12: Mode shapes for the first pair of modes: it represents the first torsional mode: (a) experimental model on the left, (b) FE model. 
TABLE 4: Summary of correlation between EMA and FEA results.

\begin{tabular}{|c|c|c|c|c|}
\hline Mode pair & EMA scaled freq. [Hz] & FEA scaled freq. $[\mathrm{Hz}]$ & Error (\%) & MAC \\
\hline 1 & 0.244 & 0.244 & 0.060 & 0.90 \\
\hline 2 & 0.254 & 0.252 & 1.055 & 0.89 \\
\hline 3 & 0.519 & 0.503 & 3.076 & 0.84 \\
\hline 4 & 0.623 & 0.612 & 1.677 & 0.96 \\
\hline 5 & 0.834 & 0.817 & 2.016 & 0.55 \\
\hline 6 & 0.849 & 0.833 & 1.934 & 0.76 \\
\hline 7 & 1.023 & 1.000 & 2.221 & 0.73 \\
\hline 8 & 1.037 & 1.019 & 1.709 & 0.73 \\
\hline 9 & 1.236 & 1.234 & 0.134 & 0.82 \\
\hline
\end{tabular}

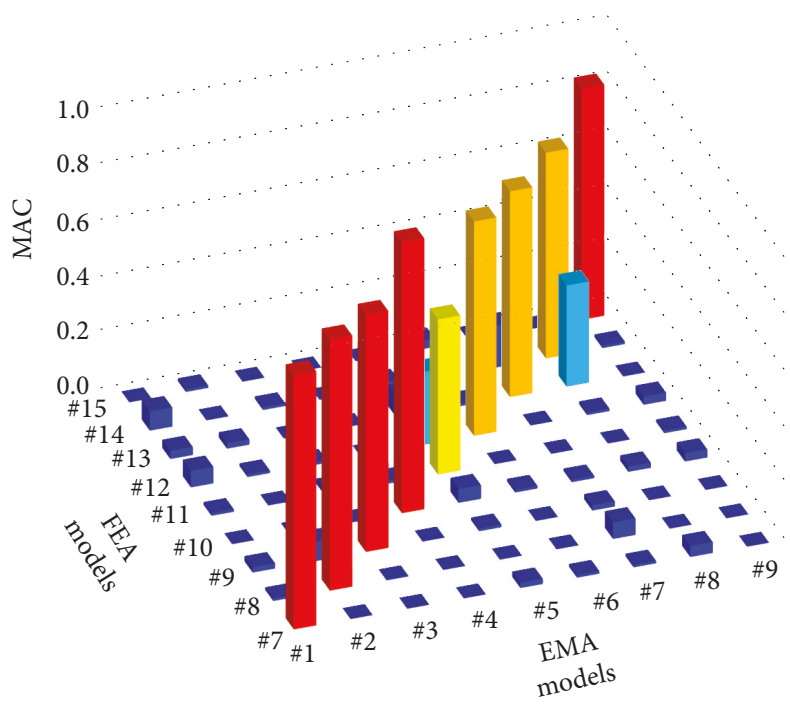

FIGURE 13: MAC matrix.

represents the sum of all the FRFs and it is worth noting that Mode\#5 is not well excited during the hammer test.

Table 4 summarizes the obtained results, evidencing the natural frequency of each mode, the absolute percentage error, between EMA and FEA, and the MAC values for each mode pair.

\section{Conclusions}

In the present paper, a correlation activity has been performed between experimental and numerical modal analyses of an aluminium rear subframe in free-free condition. In particular, a novel modelling technique of welded joints has been applied; the ACM2 approach, formulated by [16] for spot welds, has been extended to MIG. A comparison between the novel ACM2 approach and the standard rigid onedimensional method has been performed. The FEA using two different approaches lead to similar results with small differences due to stiffness contents in welding joints models. The novel ACM2 approach is used as reference for FEA results. Both an accurate FE representation of the test setup, due to the explicit modelling of testing devices, and a correct mass distribution on the structure have ensured a good agreement between EMA and FEA. Comparing the results in terms of natural frequencies, a satisfying correlation has been found; the percentage error is lower than 4 percent and it evidences a very good agreement in terms of dynamic behaviour prediction of the FE model. A detailed investigation on the modal shapes has been carried out using MAC. MAC has been formulated by [18] for evaluating the consistency of different modal vectors. The values on the main diagonal of the MAC matrix evidence a good correlation in terms of modal shapes. All the mode pairs present MAC values greater than 0.7, which represents a reference value for an acceptable correlation, evaluated by MAC. This paper represents a suitable and stable approach for an accurate validation of FE model for structural dynamic applications; evaluating the correlation between EMA and FEA for industrial purposes, it confirms the capability to predict the dynamic behaviour of the structure in the frequency range of interest.

Further improvements should be made on the experimental side in order to reduce the data noise, exciting in a proper way all the modes of the structure in the frequency range of interest. Although the correlation between EMA and FEA has been found to be good, additional developments could regard the improvement of the FE model through sensitivity and updating procedure, so as to define a more accurate numerical model for dynamic purposes. Also, better correlation could be achieved using different correlation indexes: an example is the Coordinate Modal Assurance Criterion (CoMAC) that attempts to identify which measurements contribute negatively to a low value of MAC.

\section{Conflicts of Interest}

The authors declare that there are no conflicts of interest regarding the publication of this paper.

\section{Acknowledgments}

The authors would like to thank the Ferrari NVH Team Leader Mr. Tarabra Marco and NVH Senior Engineer Mr. Roncaglia Valerio for the technical support and the guidance throughout the course of this research. The authors wish to express their sincere gratitude to Cavazzoni Luca from MilleChili Lab for the invaluable assistance in the final review of the paper. 


\section{References}

[1] M. Baker, "Review of test/analysis correlation methods and criteria for validation of finite element models for dynamic analysis," in Proceedings of the 10th International Modal Analysis Conference, San Diego, Calif, USA, February 1992.

[2] M. Imregun and W. J. Visser, "A review of model updating techniques," Shock and Vibration Digest, vol. 23, no. 1, pp. 141-162, 1990.

[3] M. I. Friswell and J. E. Mottershead, Finite Element Model Updating in Structural Dynamics, vol. 38 of Solid Mechanics and Its Applications, Kluwer Academic, Dordrecht, Netherlands, 1995.

[4] M. Brughmans, J. Leuridan, T. Van Langenhove, and F. Turgay, "Validation of automotive component FE models by means of test-analysis correlation and model updating techniques," SAE Technical Paper 1999-01-1797, 1999.

[5] S. Jambovane, D. Kalsule, and S. Athavale, "Validation of FE models using experimental modal analysis," SAE Technical Paper 2001-26-0042, 2001.

[6] D. Siano, M. Viscardi, P. Napolitano, and M. A. Panza, "Experimental/FE numerical correlation of a composite sandwich panel of a high-speed train," in Proceedings of the 10th International Conference on Applied and Theoretical Mechanics (MECHANICS '14), pp. 978-960, Salerno, Italy, 2014.

[7] L. Splendi, L. D’Agostino, A. Baldini, L. Castignani, F. Pellicano, and M. Pinelli, "Simplified modeling technique for damping materials on light structures: experimental analysis and numerical tuning," in Proceedings of the ASME International Mechanical Engineering Congress and Exposition (IMECE '13), San Diego, Calif, USA, November 2013.

[8] C. Schedlinski, F. Wagner, K. Bohnert et al., “Test-based computational model updating of a car body in white," Sound and Vibration, vol. 39, no. 9, pp. 19-23, 2005.

[9] S. Mariano, M. da Silva, A. de Costa Moreira et al., "Modal correlation of an aerospace structure," SAE Technical Paper 2006-01-2786, 2006.

[10] C. Azoury, A. Kallassy, B. Combes, I. Moukarzel, and R. Boudet, "Experimental and analytical modal analysis of a Crankshaft," IOSR Journal of Engineering, vol. 2, no. 4, pp. 674-684, 2012.

[11] B. J. Schwarz and M. H. Richardson, "Experimental Modal Analysis," in Proceedings of the CSI Reliability Week, Orlando, Fla, USA, 1999.

[12] B. Peeters, P. Guillaume, H. Van der Auweraer, B. Caubergue, P. Verboven, and J. Leuridan, "Automotive and aerospace applications of the PolyMAX modal parameter estimation method," in Proceedings of the 22nd International Modal Analysis Conference, pp. 17-21, Dearborn, Mich, USA, January 2004.

[13] M. Aygül, Fatigue analysis of welded structures using the finite element method [Licentiate of Engineering thesis], Department of Civil and Environmental Engineering, Chalmers University of Technology, Gothenburg, Sweden, 2012.

[14] K. Pal and D. L. Cronin, "Static and dynamic characteristics of spot welded sheet metal beams," Journal of engineering for industry, vol. 117, no. 3, pp. 316-322, 1995.

[15] J. Backhans and A. Cedas, A finite element model of spot welds between non-congruent shell meshes calculation of stresses for fatigue life prediction [M.S. thesis], Volvo Car Corporation, Gothenburg, Sweden, Analysis report, 93841-2000, 2000.

[16] D. Heiserer, M. Charging, and J. Sielaft, "High performance, process oriented, weld spot approach," in Proceedings of the 1st MSC Worldwide Automotive User Conference, Munich, Germany, September 1999.

[17] M. Palmonella, M. I. Friswell, J. E. Mottershead, and A. W. Lees, "Finite element models of spot welds in structural dynamics: review and updating," Computers and Structures, vol. 83, no. 8-9, pp. 648-661, 2005.

[18] R. J. Allemang and D. L. Brown, "Correlation coefficient for modal vector analysis," in Proceedings of the 1st International Modal Analysis Conference \& Exhibit, pp. 110-116, Orlando, Fla, USA, November 1982.

[19] R. J. Allemang, "The modal assurance criterion (MAC): twenty years of use and abuse," in Proceedings of the IMAC 20, The International Modal Analysis Conference, pp. 397-405, Los Angeles, Calif, USA, 2002. 


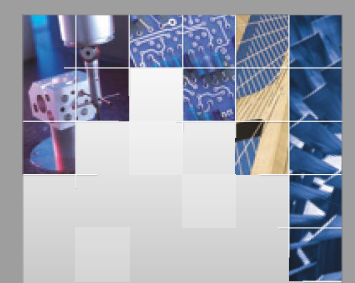

\section{Enfincering}
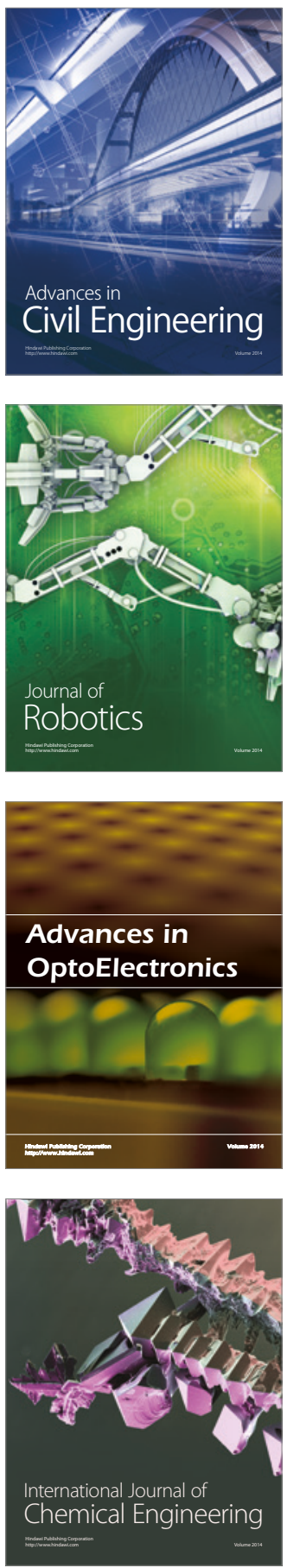

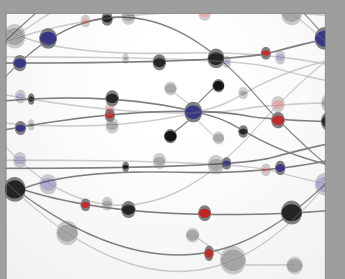

The Scientific World Journal

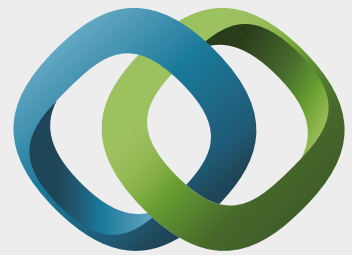

\section{Hindawi}

Submit your manuscripts at

https://www.hindawi.com
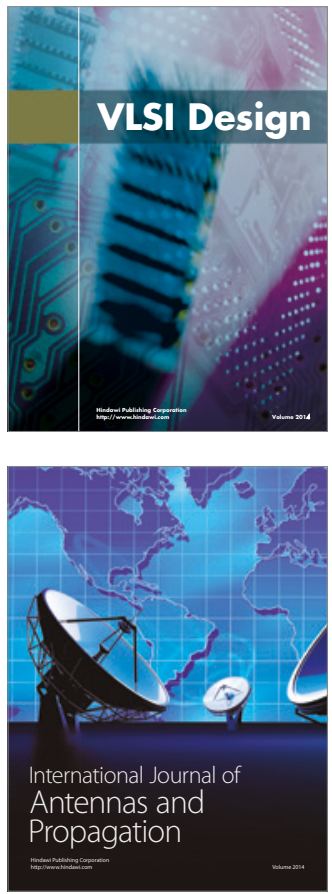

\section{Rotating}

Machinery
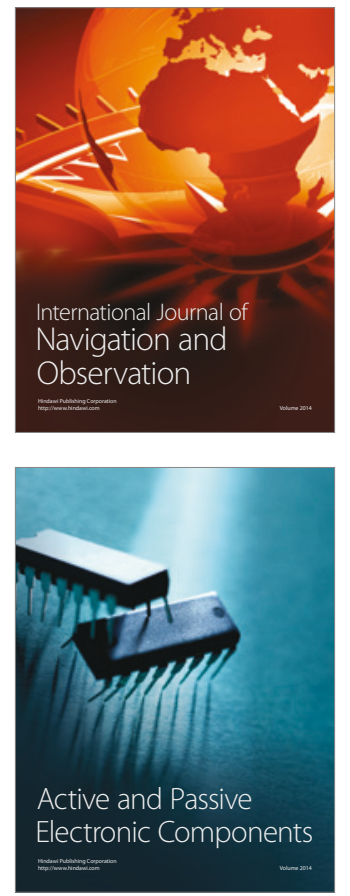
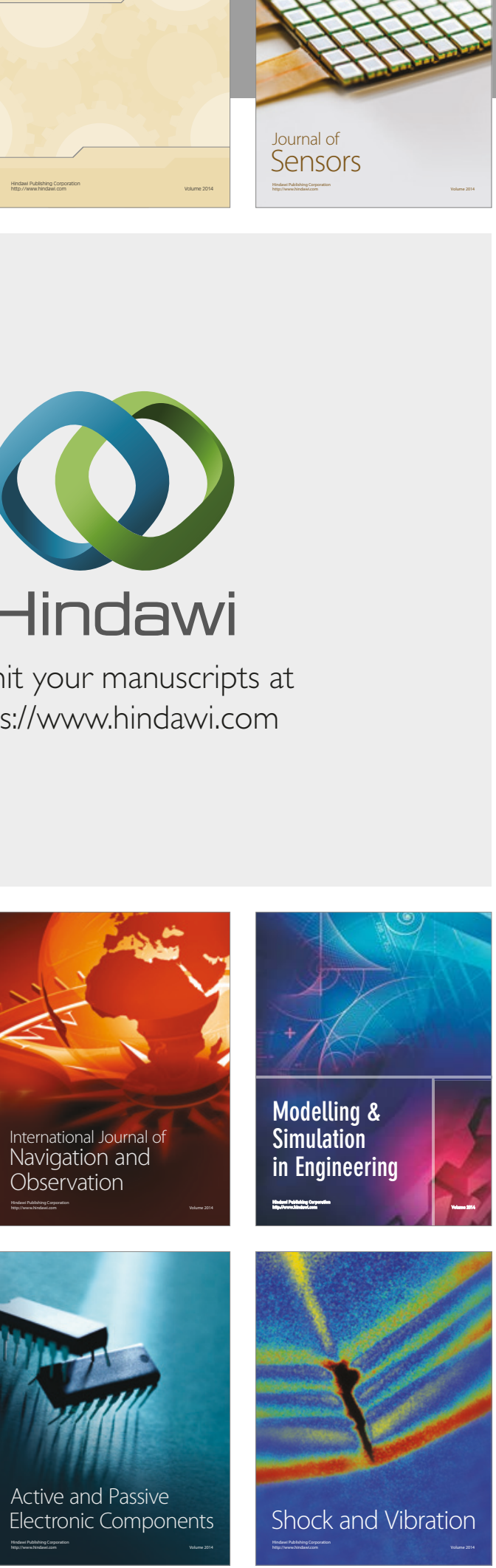
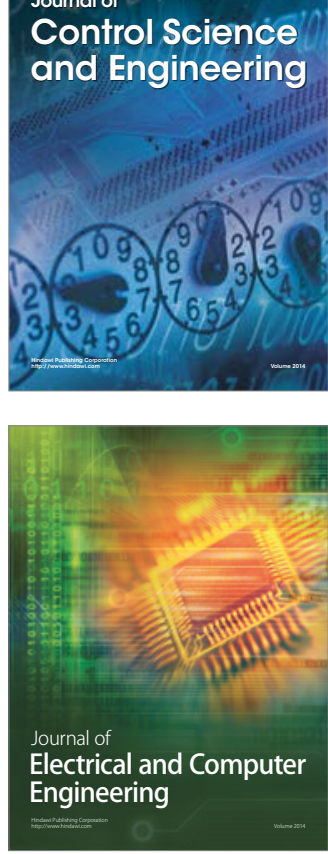

Distributed

Journal of

Control Science

and Engineering
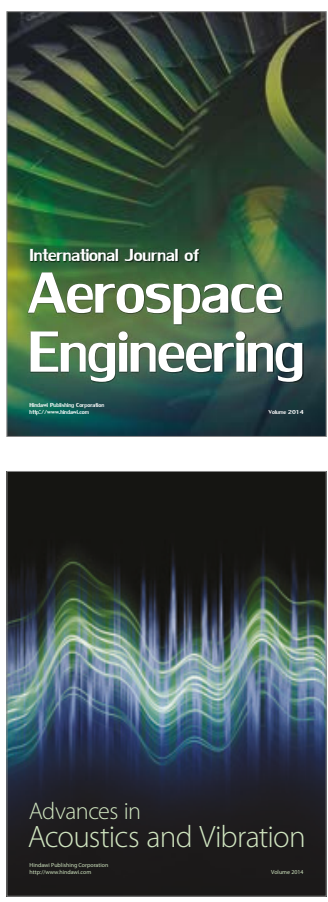

Sensor Networks 